\section{Commentary: Thoracoabdominal aneurysmectomy: Operative steps for Crawford extent II repair: The devil is in the detail}

\author{
Jean Porterie, MD, and François Dagenais, MD
}

Although open surgical repair remains the gold standard for treatment of thoracoabdominal aortic aneurysms (TAAA), it still carries significant mortality and serious adverse events, justifying efforts to protect the spinal cord and the visceral organs from ischemia-related complications. Lopez-Marco and colleagues ${ }^{1}$ report a very interesting step-by-step illustration of their current approach to open extent II TAAA repair. The authors routinely use sequential aortic crossclamping under left heart bypass with mild passive hypothermia $\left(34^{\circ} \mathrm{C}\right)$, selective visceral and medullar isothermic blood perfusion, as well as renal protection through intermittent administration of cold Custodiol solution (Essential Pharmaceuticals, LLC, Durham, NC) in the renal arteries, cerebrospinal fluid drainage, monitoring of motor evoked potentials, and near-infrared spectrometry of cerebral, paraspinal, and lower limbs oxygen saturations. They advocate for individual visceral artery reimplantation using a branched graft and when possible a selective reattachment of 1 or more pairs of the lower thoracic intercostal arteries using a separate $10-$ to $12-\mathrm{mm}$ graft. The authors conclude that favorable early outcomes and a durable repair can be achieved at experienced, high-volume centers.

Despite improvements in neuroprotective measures, spinal cord injury remains the Achilles heel of open and endovascular TAAA repairs; they are reported in up to $31 \%$ of

\footnotetext{
From the Department of Cardiac Surgery, Institut Universitaire de Cardiologie et de Pneumologie de Québec, Université Laval, Québec, Québec, Canada.

Disclosures: The authors reported no conflicts of interest.

The Journal policy requires editors and reviewers to disclose conflicts of interest and to decline handling or reviewing manuscripts for which they may have a conflict of interest. The editors and reviewers of this article have no conflicts of interest.

Received for publication Aug 14, 2020; revisions received Aug 14, 2020; accepted for publication Aug 14, 2020; available ahead of print Aug 19, 2020.

Address for reprints: François Dagenais, MD, FRCSC, Institut Universitaire de Cardiologie et de Pneumologie de Québec, 2725 Chemin Sainte-Foy, Québec, QC, Canada G1V 4G5 (E-mail: francois.dagenais@chg.ulaval.ca).

JTCVS Techniques 2020;3:41-2

2666-2507

Copyright (C) 2020 The Authors. Published by Elsevier Inc. on behalf of The American Association for Thoracic Surgery. This is an open access article under the CC BY-NCND license (http://creativecommons.org/licenses/by-nc-nd/4.0/).

https://doi.org/10.1016/j.xjtc.2020.08.044
}

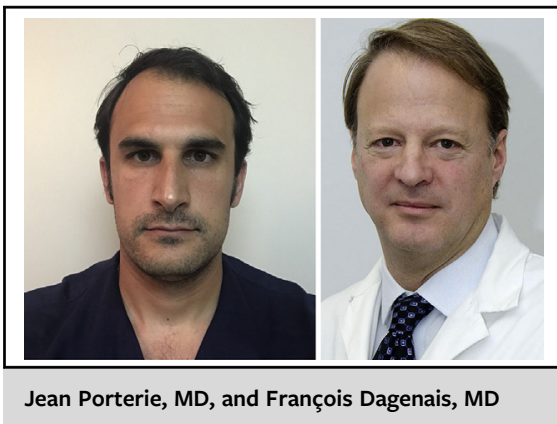

\author{
CENTRAL MESSAGE \\ A multimodal, multidisciplinary \\ strategy is pivotal in open repairs \\ of extent type II thoracoabdo- \\ minal aortic aneurysms to pre- \\ vent spinal cord and the visceral \\ organs from ischemia-related \\ complications.
}

type II TAAA repairs. The coverage of potentially relevant intercostal arteries during thoracic endovascular aortic repair, with relatively low rates of spinal cord injury, suggests that the mechanism is not exclusively anatomical but multifactorial. Thus, a multimodal spinal cord protection strategy is pivotal and should integrate preservation of the patient's functional collateral network, hemodynamic stability, and reduction of ischemia duration. ${ }^{2}$

This paradigm is well illustrated by the valuable work of the authors, but further considerations should be outlined. Necessity of selective intercostal artery reimplantation-especially in presence of vigorous back bleeding - remains debated. In expert hands, intraoperative motor evoked potentials monitoring during sequential crossclamping may provide information on which vessels are essential to reimplant, although the authors mention not basing their decision on its results. In addition, prolonged clamping of the left subclavian artery should be avoided. Finally, in case of TAAA extending to the arch, a 1-stage procedure with extensive aortic replacement under deep hypothermic circulatory arrest is associated with high mortality and neurologic risk. In such cases, a 2-stage approach using initially either a hybrid graft or a standard elephant trunk procedure followed by the TAAA repair may be advisable to lower the overall morbidity/ mortality. $^{3}$

As supported by the authors, the outcomes of TAAA open repair seem to be widely related to institutional experience. 
In a recent study including more than 14,000 patients from the US Nationwide Inpatient Sample, in-hospital mortality was at least 1.5 times higher at low- and medium-volume centers compared with high-volume $(\geq 12$ cases/year) centers, in both the elective and urgent cases. TAAA volume was associated with increased use of distal aortic perfusion. ${ }^{4}$ Experienced teams are more prone to perform standardized perioperative care and include multidisciplinary management. Efforts to make open TAAA repair a more standardized and reproducible procedure are to be encouraged and justify establishment of dedicated multidisciplinary teams, especially in the rapidly emerging era of branch graft endovascular therapy.

\section{References}

1. Lopez-Marco A, Adams B, Oo A. Thoracoabdominal aneurysmectomy: operative steps for Crawford extent II repair. J Thorac Cardiovasc Surg Tech. 2020;3:25-36.

2. Etz CD, Weigang E, Hartert M, Lonn L, Mestres CA, Di Bartolomeo R, et al. Contemporary spinal cord protection during thoracic and thoracoabdominal aortic surgery and endovascular aortic repair: a position paper of the vascular domain of the European Association for Cardio-Thoracic Surgery. Eur J Cardiothorac Surg. 2015;4:943-57.

3. Czerny M, Schmidli J, Adler S, van den Berg JC, Bertoglio L, Carrel T, et al. Current options and recommendations for the treatment of thoracic aortic pathologies involving the aortic arch: an expert consensus document of the European Association for Cardio-Thoracic surgery (EACTS) and the European Society for Vascular Surgery. Eur J Cardiothorac Surg. 2019;55:133-62.

4. Polanco AR, D’Angelo AM, Shea NJ, Allen P, Takayama H, Patel VI. Increased hospital volume is associated with reduced mortality after thoracoabdominal aortic aneurysm repair. J Vasc Surg. May 27, 2020 [Epub ahead of print]. 